\title{
Shrinking II — The Distortion of the Area Distance-Redshift Relation in Inhomogeneous Isotropic Universes
}

\author{
Nazeem Mustapha, ${ }^{*}$ B.A. Bassett,${ }^{\dagger}$ Charles Hellaby, ${ }^{\ddagger}$ and G.F.R. Ellis ${ }^{\S}$ \\ Department of Mathematics and Applied Mathematics, University of Cape Town, Rondebosch, 7700, South Africa
}

\begin{abstract}
This paper and the others in the series [1, 2] challenge the standard model of the effects of gravitational lensing on observations at large distances. We show that due to the cumulative effect of lensing, areas corresponding to an observed solid angle can be quite different than would be estimated from the corresponding Friedmann-Lemaitre model, even when averaged over large angular scales. This paper concentrates on the specific example of spherically symmetric but spatially inhomogeneous dust universes, the Lemaître-Tolman-Bondi models, and shows that radial lensing significantly distorts the area distance-redshift and density-redshift relations in these exact solutions compared with the standard ones for Friedmann-Lemaitre models. Thus inhomogeneity may introduce significant errors into distance estimates based on the standard FL relations, even after all-sky averaging. In addition a useful new gauge choice is presented for these models, solving the problem of locating the past null cone exactly.
\end{abstract}

04.20.Cv, 95.30.Sf, 98.60.Eg, 98.60.Gi, 98.80.Es

Short Title: Shrinking II

Submitted to Class. Q. Grav. March 1997

\section{INTRODUCTION AND GENERAL ARGUMENTS}

Our aim in this series of papers is to show that when analyzing observations at large redshift in the real universe, the assumption of an area distance corresponding to that of a best-fit Friedmann-Lemaitre (FL) model — that is, one with a matching averaged matter density - may not be a good approximation, even when averaging over large angular scales. This claim is important because of the ubiquitous use of FL models in studies of number counts versus redshift and area distance versus redshift.

The first paper in the series (paper I) [1] gave general arguments for this thesis. It was explained there that whilst the Dyer-Roeder distance [3] is generally regarded as a good approximation for ray bundles moving between high-density clusters of matter, resulting in a de-focussing relative to the comparable FL model, matter moving near or through higher density regions is more focused than in the FL model, so resulting in a compensating effect. It is commonly believed [4, 5] that an exact cancellation between the two effects takes place when one averages over large angular scales containing both high and low density regions - so the FL area distance is the correct one on these scales. However, it was pointed out in [1] that, after caustics have formed through the focussing of light rays by the high density regions, these light rays too are rapidly diverging so that sufficiently far down the past light cone all light rays will be diverging relative to the comparable FL model. Consequently there is good reason to question the general opinion in this matter - we find that in fact 'shrinking' takes place. $I^{2}$ It is often argued that photon conservation excludes this possibility, but these arguments are based on incorrect assumptions about the geometry.

\footnotetext{
*email: nazeem@maths.uct.ac.za

${ }^{\dagger}$ email: bruce@stardust.sissa.it

${ }^{\ddagger}$ email: cwh@maths.uct.ac.za

$\S$ email: ellis@maths.uct.ac.za

${ }^{1}$ We follow recent moves to use 'Friedmann-Lemaitre' to describe the dynamics of and the application of the Einstein field equations to the standard model, and 'Robertson-Walker' to describe its metric and geometry.

${ }^{2}$ The term shrinking [6] describes the following. If at some redshift the area distance is greater in the inhomogeneous universe than in the best-fit or background FL model, scales at that redshift will be underestimated if the FL area distance is used to calculate the size of objects from their measured angular size, and so will have 'shrunk' relative to their actual scales in the more realistic model. Put another way, if a sphere at given $z$ about the observer has a larger total area in the inhomogeneous model, an object of fixed size will subtend a smaller solid angle on the sky, and so be shrunk. (See [1] for more discussion.)
} 
Given this feature, even when there is only weak lensing, 3 and caustics have not formed, it is not obvious that the FL area distance-redshift relation is an accurate description.

The aim of this paper is to construct an exact inhomogeneous model and its FL approximation, and compare the area distance-redshift and density-redshift relations in the two. To do so we examine Lemaître-Tolman-Bondi (LTB) spherically symmetric dust solutions [7] 9], where exact integrations of the field equations are available for the past light cone of observers at the central position. Although the lensing that occurs for this central observer is purely radial, we find the inhomogeneity has a tangible effect on observational relations. If In the particular case examined, because of the spherical symmetry about the observer, the effect in any specific direction will not be compensated by an opposite effect in another direction - on the contrary, the effect is uniform because it is the same in all directions, and will occur on large as well as small angular scales. However unlike the previous paper [1], this will not be associated with the formation of caustics.

The failure of the FL-like area distance-redshift assumption will thus have been shown to occur for observers at a very special position in a family of high-symmetry space-times. Undoubtedly this is not the kind of situation the authors of the papers mentioned above, claiming the effect does not occur, had in mind. However the usual statements of this effect contain no clauses that exclude this situation: the result is supposed to hold in all cases when the 'lumpy' universe is reasonably close to an FL model, and observations are averaged over the sky; their arguments do not exclude the situation envisaged here where the observer is at the centre of a spherically symmetric inhomogeneity. Indeed, a spherically symmetric model may be regarded as describing data that has been averaged over the whole sky, but not over distance.

Our example thus confirms the claims of paper I, in the setting of particular exact inhomogeneous solutions of the Einstein Field Equations. It does not generically establish the magnitude of the effect, precisely because the high-symmetry geometry considered here precludes formation of caustics and the consequent fractal-like structure of the real light cone. Paper III [2] will provide confirmation of the overall shrinking effect due to caustics, and attempt more realistic estimates of its magnitude than those given in paper I, which used simple analytic formulae for this purpose.

In developing the results of this paper, we solve one of the problems that has made analysis of observations in Lemaitre-Tolman-Bondi solutions difficult, namely the problem of precisely locating the past light cone of the chosen central event $\mathrm{P}$, by use of a special choice of radial coordinate that ensures a very simple form for the past light cone of $\mathrm{P}$ in these inhomogeneous space-times. This technical development has other uses in terms of analysing observational relations in these models.5 Our approach is complementary to that of Kurki-Suonio and Liang [12], who did numerical calculations of observational relations in a hyperbolic LTB model derived from an $\Omega=0.1$ FL model plus some overdensities.

\section{PROGRAMME}

We select the simplest inhomogeneous fluid solution of the Einstein Field Equations; the Lemaitre-Tolman-Bondi (LTB) model which is spherically symmetric, but radially inhomogeneous, with a dust equation of state.

The question we are raising is whether the area of an averaged wavefront we receive at our observatory in an inhomogeneous universe is the same as the area of a wavefront in a smoothed version of that universe. To clarify this issue our strategy is to:

- Select the most natural generalisation of the Einstein- de Sitter models commonly used in studies of observations (i.e. a parabolic LTB model) and describe data on the null cone.

- Find the FL limit of this inhomogeneous universe in an appropriate coordinate system.

- Average the lumpy universe in a natural way and fit it correctly to a FL model.

- Compare area distances in the lumpy universe and its smoothed average.

\footnotetext{
${ }^{3}$ We refer to 'lensing' when the light rays are different than they would be in the background FL model. Thus weak lensing implies a change of apparent positions and alteration in the usual distance relations, usually combined with image distortions, but not necessarily multiple imaging.

${ }^{4}$ Radial lensing is a spherically symmetric distortion of the null cone compared with an FL model, resulting in a uniform delay of the wavefront. There is no image distortion, no dependence of magnification or time delay on direction, and no multiple imaging, but our results show its effects are observable.

${ }^{5}$ For a slightly different analysis of LTB spacetimes, based on null cone coordinates, see [10], and for a consideration of observations away from the centre of symmetry see [11.
} 


\section{A. The Inhomogeneous Model}

\section{The Integrated Field Equations}

We choose the parabolic LTB model which is the natural generalisation of the $\Omega=1$ dust FL model. This model is characterised by the mass within comoving radius $r, M(r)$, and the 'bang-time' function $t_{B}(r)$ describing the locus of the initial spatial hypersurface (that is, the local time of the big bang). The two arbitrary functions represent a physical freedom and a coordinate freedom, e.g. $t_{B}(M)$ and $M(r)$, respectively.

In normalised comoving coordinates the metric after solving the off-diagonal EFE is

$$
d s^{2}=-d t^{2}+\left(R^{\prime}\right)^{2} d r^{2}+R^{2}\left(d \theta^{2}+\sin ^{2} \theta d \Phi^{2}\right)
$$

where $R^{\prime}(t, r)=\partial R(t, r) / \partial r$.

The time curves are irrotational and, for comoving dust $(p=0)$, are necessarily geodesics because of momentum conservation. The spatial sections are flat because if we choose $r=R\left(t_{0}, r\right)$ then $R^{\prime}\left(t_{0}, r\right)=1$ and we find that the 3-spaces have metric $d \sigma^{2}=d r^{2}+r^{2} d \Omega^{2}$ and hence are flat.

The areal radius, $R=R(t, r)$ in the Lemaitre-Tolman-Bondi metric, is the area of the intersection of our past null cone with past spacelike time surfaces (in this case spheres). In the parabolic case $R$ is given explicitly by the solution to the equation of motion

$$
\dot{R}(t, r)=\sqrt{\frac{M(r)}{R(t, r)}}
$$

obtained from the 11, 22 and 33 components of the EFE, where 'denotes the derivative with respect to $t$; i.e.

$$
R(t, r)=\left[\frac{9 M(r)}{2}\left(t-t_{B}(r)\right)^{2}\right]^{1 / 3}
$$

where $t$ is cosmic time whilst $M$ and $t_{B}$ are both functions of coordinate radius $r$ only. It follows immediately that

$$
R^{\prime}(t, r)=\frac{3}{2 R^{2}(t, r)}\left[M^{\prime}(r)\left(t-t_{B}(r)\right)^{2}-2 M(r)\left(t-t_{B}(r)\right) t_{B}^{\prime}(r)\right]
$$

where' denotes the derivative with respect to radial coordinate $r$.

The 00 field equation gives

$$
4 \pi \rho(t, r)=\frac{M^{\prime}(r)}{R^{2}(t, r) R^{\prime}(t, r)}
$$

\section{The Solution on the Null Cone}

Since we are interested in observations on the null cone we must project onto it by specifying the unique relationship between $r$ and $t$. On radial null geodesics, $d s^{2}=0=d \theta^{2}=d \Phi^{2}$, so from (1) , if the past light cone of the event $\left(t=t_{0}, r=0\right)$ is given by $t=\hat{t}(r)$, then that light cone is described by

$$
d t= \pm R^{\prime}(\hat{t}(r), r) d r
$$

The coordinate freedom in the LTB metric is a rescaling of the radial coordinate $r \rightarrow \tilde{r}=\tilde{r}(r)$. If we choose $r$ so that

$$
R^{\prime}(\hat{t}(r), r)=1
$$

then on the past light cone $d t=-d r$, so that the incoming light rays at the event $\left(t=t_{0}, r=0\right)$ are given by

$$
\hat{t}(r)=t_{0}-r .
$$

So this gauge choice, in contrast to other work done on observations in the LTB model, locates the null cone of the observer at one instant of time, in its simplest possible form, and makes our programme analytically solvable. On this light cone, putting (8) in (4) and using (7), 


$$
R^{\prime}(\hat{t}(r), r)=\frac{3}{2 R^{2}(\hat{t}(r), r)}\left[M^{\prime}(r) \tau(r)^{2}+2 M(r) \tau(r) \tau^{\prime}(r)+2 M(r) \tau(r)\right]=1
$$

where we have defined

$$
\tau(r)=t_{0}-r-t_{B}(r) .
$$

The function $\tau(r)$ can be interpreted as proper time from the bang surface to our past null cone along the particle worldlines. We can set $t_{0}$ to be the time since the bang at the observer $(r=0)$ by choosing $t_{B}(0)=0$ (so $\left.\tau(0)=t_{0}\right)$.

It is important to realise that evaluating $R^{\prime}(t, r)$ on the null cone $t=\hat{t}(r)$ is not the same as differentiating $\hat{R}(r)=R(\hat{t}(r), r)$ with respect to $r$. In fact, by evaluating (3) on the null cone, $\hat{R}$ is given by

$$
\hat{R}=R(\hat{t}(r), r)=\left[\frac{9 M(r)}{2} \tau(r)^{2}\right]^{1 / 3}
$$

which means that its derivative is given by

$$
\frac{d \hat{R}}{d r}=\frac{d}{d r}[R(\hat{t}(r), r)]=\frac{3}{2 R^{2}(\hat{t}(r), r)}\left[M^{\prime}(r) \tau(r)^{2}+2 M(r) \tau(r) \tau^{\prime}(r)\right] .
$$

Combining the above equation with the constraint (9) gives a first order differential equation for $\hat{R}$.

$$
\frac{d \hat{R}(r)}{d r}+\frac{3 M(r) \tau(r)}{\hat{R}(r)^{2}}=1
$$

In summary, with our choice of coordinates we have recast the flat LTB model in a form that allows us to locate the past null cone with ease. This has left us with one physical freedom to choose an arbitrary function of $r$. We could choose $\tau$ (or $M$ ) and substitute (11) into (13). Solution of this differential equation would determine $\hat{R}$ and thus any other quantity. If we instead decide to choose $\hat{R}$, that is, the area of the wavefront, then the model is trivially and fully specified by (11) and (13). It follows that

$$
\tau(r)=\frac{2 \hat{R}(r)}{3} /\left(1-\frac{d \hat{R}(r)}{d r}\right)
$$

and

$$
M(r)=\frac{\hat{R}(r)}{2}\left(1-\frac{d \hat{R}(r)}{d r}\right)^{2} \quad(M(0)=0) .
$$

To obtain the results of the next section, we will fix the geometry by choosing $\hat{R}$. This will then determine $\tau(r)$ (equivalently $\left.t_{B}(r)\right)$ and $M(r)$ by the above two equations. The flat LTB model will thus be fully specified in these coordinates and one could then propagate the data off the null cone by the comoving assumption.

The density on the null cone $\hat{\rho}(r)$ is found by evaluating (5) on the null cone:

$$
4 \pi \hat{\rho}(r)=\frac{M^{\prime}(r)}{\hat{R}(r)^{2}}
$$

and its value at the origin depends on the time as characterised by the Hubble constant;

$$
\Omega=\frac{4 \pi \rho}{H^{2}}=1 \Rightarrow \rho_{0}=\frac{H_{0}^{2}}{4 \pi}, t_{0}=\frac{2}{3 H_{0}} .
$$

\footnotetext{
${ }^{6}$ Since measurements of the Hubble constant are taken at about $z<1$, we can take this to determine the age of the universe, $t_{0}$, at the central observer.
} 


\section{Redshifts}

It is of some importance that we state the relevant quantities in terms of redshifts. To do this, we use the fact that in the geometric optics limit, for two light rays emitted on the worldline at $r_{e m}$ with time interval $\delta t_{e m}=$ $t^{+}\left(r_{e m}\right)-t^{-}\left(r_{e m}\right)$ and observed on the central worldline with time interval $\delta t_{o b}=t^{+}(0)-t^{-}(0)$

$$
1+z=\frac{\delta t_{o b}}{\delta t_{e m}} .
$$

The past radial null geodesics are given by

$$
d t=-R^{\prime}(t, r) d r
$$

so for an observer on a nearby worldline, the time interval changes by

$$
d(\delta t)=d t^{+}-d t^{-}=\left[R^{\prime}\left(t^{-}, r\right)-R^{\prime}\left(t^{+}, r\right)\right] d r=-\frac{\partial}{\partial t}\left[R^{\prime}(t, r)\right] \delta t d r .
$$

Thus

$$
d \ln \delta t=-\frac{\partial}{\partial t}\left[R^{\prime}(t, r)\right] d r
$$

which means that the redshift, given by (18), is

$$
\ln (1+z)=\int_{0}^{r_{e m}} \dot{R}^{\prime}(\hat{t}, r) d r
$$

where $\hat{t}(r)$ is the equation of the null cone.7 To calculate $\dot{R}^{\prime}(t, r)$ we differentiate (3) with respect to $t$

$$
\dot{R}^{\prime}=\frac{\dot{R}}{3}\left[\frac{t_{B}^{\prime}}{t-t_{B}}+\frac{M^{\prime}}{M}\right], \quad \dot{R}=\left[\frac{4 M}{3\left(t-t_{B}\right)}\right]^{1 / 3} .
$$

Since $\hat{t}(r)=t_{0}-r$ when we choose $R^{\prime}=1$ on the null cone, $\dot{R}^{\prime}(\hat{t}, r)$ is given by

$$
\dot{R}^{\prime}(\hat{t}, r)=\frac{1}{3}\left[\frac{4 M}{3 \tau}\right]^{1 / 3}\left[\frac{M^{\prime}}{M}-\frac{1+\tau^{\prime}}{\tau}\right]
$$

After some manipulation of the above expression substituted into (19), we find that

$$
\ln (1+z)=\left(\frac{4 M}{3 \tau}\right)^{1 / 3}-\frac{1}{3} \int_{0}^{r_{e m}}\left(\frac{4 M}{3 \tau^{4}}\right)^{1 / 3} d r
$$

Using (14) and (15) this equation may be written as

$$
\ln (1+z)=\left(1-\frac{d \hat{R}}{d r}\right)-\frac{1}{2} \int_{0}^{r_{e m}}\left(1-\frac{d \hat{R}}{d r}\right)^{2} / \hat{R} d r
$$

so we can now determine the redshift-area distance relation.

\footnotetext{
${ }^{7}$ The standard formula $1+z=\left(u^{\mu} k_{\mu}\right)_{e m} /\left(u^{\mu} k_{\mu}\right)_{o b}$ is not useful in this gauge since $k^{\mu}=\left(R^{\prime},-1,0,0\right)=(1,-1,0,0)$ is not geodesic, though it is tangent to the past null cone.
} 


\section{B. The Friedmann-Lemaître Limit}

The characterisation of the FL limit is that the bang time surface is simultaneous. So $t_{B}(r)=t_{B \mathrm{FL}}=$ constant; from whence

$$
R_{\mathrm{FL}}(t, r)=\left[\frac{9}{2} M_{\mathrm{FL}}(r)\left(t-t_{B_{\mathrm{FL}}}\right)^{2}\right]^{1 / 3}, \quad R_{\mathrm{FL}}^{\prime}(t, r)=\frac{3}{2 R_{\mathrm{FL}}^{2}} M_{\mathrm{FL}}^{\prime}(r)\left(t-t_{B_{\mathrm{FL}}}\right)^{2} .
$$

The freedom left here in $M_{\mathrm{FL}}(r)$ is just essentially the coordinate freedom, corresponding to the freedom of choice of $r$. The above relations determine the FL density

$$
\rho_{\mathrm{FL}}(t)=\frac{1}{6 \pi\left(t-t_{B \mathrm{FL}}\right)^{2}}
$$

which is spatially homogeneous as required, unaffected by $M_{\mathrm{FL}}(r)$. It is usual to set $t_{B \mathrm{FL}}=0$. We do not have a freedom to rescale the density by a constant because this is the critical density case.

As we would eventually like to compare our LTB model as chosen above to an underlying FL model, it is appropriate to write the FL limit in the same kind of coordinate system. Consider light rays coming in to the event $\left(t=t_{1}, r=0\right)$ in a FL model. When we choose coordinates for which $R_{\mathrm{FL}}^{\prime}(t, r)=1$ on the null cone, the past null cone can be located by $\hat{t}=t_{1}-r-t_{B \mathrm{FL}}=t_{1}-r$. (We use $t_{1}$ rather than $t_{0}$ here, as we will need to distinguish LTB and FL values later on.) As a limit of the flat LTB model in these coordinates, the FL form of $M(r)$ is obtained from setting $\tau=t_{1}-r$ in (9). This yields

$$
\begin{aligned}
& M_{\mathrm{FL}}=6\left[t_{1}^{1 / 3}-\left(t_{1}-r\right)^{1 / 3}\right]^{3} \quad\left(M_{\mathrm{FL}}(0)=0\right) \\
& \hat{R}_{\mathrm{FL}}=3\left[t_{1}^{1 / 3}-\left(t_{1}-r\right)^{1 / 3}\right]\left(t_{1}-r\right)^{2 / 3} \quad\left(R_{\mathrm{FL}}(0)=0\right) \text {. }
\end{aligned}
$$

We note that this in conjunction with 24 implies that

$$
R_{\mathrm{FL}}(t, r)=3\left[t_{1}^{1 / 3}-\left(t_{1}-r\right)^{1 / 3}\right] t^{2 / 3} \quad, \quad R_{\mathrm{FL}}^{\prime}=\frac{3 M_{\mathrm{FL}}^{\prime}}{2 R_{\mathrm{FL}}^{2}} t^{2}=\frac{t^{2 / 3}}{\left(t_{1}-r\right)^{2 / 3}} .
$$

The RW metric that results is, from (28) and (11),

$$
d s^{2}=-d t^{2}+t^{4 / 3}\left\{\frac{1}{\left(t_{1}-r\right)^{4 / 3}} d r^{2}+9\left[t_{1}^{1 / 3}-\left(t_{1}-r\right)^{1 / 3}\right]^{2} d \Omega^{2}\right\} .
$$

These coordinates are singular at the particle horizon, $r=t_{1}$ (when the past null cone of $t=t_{1}$ runs into the initial singularity). Thus they are valid for $0 \leq r<t_{1}$. The FL redshift-distance formula can be obtained by inserting the FL forms of $M(r)$ and $\tau(r)$ into equation (22). That is

$$
z(r)=\left(\frac{t_{1}}{t_{1}-r}\right)^{2 / 3}-1 \quad \Longleftrightarrow \quad r(z)=t_{1}\left[\frac{(1+z)^{3 / 2}-1}{(1+z)^{3 / 2}}\right] .
$$

\section{Averaging and Fitting}

We want to compare and contrast total areas of wavefronts at given redshifts of an inhomogeneous model to that of the corresponding FL model of density equal to the inhomogeneous density perfectly smoothed. This must be done with respect to the inhomogeneous metric because physically the smoothing does not occur.

Perhaps the crucial part of our analysis is that we ensure that we compare with the FL model with the correct average density. We define the average or background FL model to be the one that matches on at the particle horizon where $\tau=0, r=r_{\Sigma}$, using the Darmois-Israel boundary conditions [13,14]. Matching first and second fundamental forms of this timelike (comoving) boundary surface $\Sigma$ gives

$$
\left.R_{\mathrm{LTB}}\right|_{\Sigma}=\left.R_{\mathrm{FL}}\right|_{\Sigma}
$$

and the background model must be parabolic if the inhomogeneous one is; or vice versa. 
The matching must hold over all of $\Sigma$; that is, at all times — so

$$
\left.\dot{R}_{\mathrm{LTB}}\right|_{\Sigma}=\left.\dot{R}_{\mathrm{FL}}\right|_{\Sigma}
$$

and thus, by (2),

$$
\left.M_{\mathrm{LTB}}\right|_{\Sigma}=\left.M_{\mathrm{FL}}\right|_{\Sigma}
$$

Thus it is sufficient to match the masses at $\Sigma$, and synchronise the starting times (bang times) when $\left.R_{\mathrm{LTв}}\right|_{\Sigma}=0=$ $\left.R_{\mathrm{FL}}\right|_{\Sigma}$. In general, we do not expect the FL radial coordinate on $\Sigma\left(\left.r_{\mathrm{FL}}\right|_{\Sigma}\right)$ to be the same as the LTB one there $\left(\left.r_{\mathrm{LTB}}\right|_{\Sigma}=r_{\Sigma}\right)$ since the coordinate condition $\hat{R}^{\prime}=1$ holds on the null cone, whose locus is model dependent.

For a parabolic LTB model with metric (1) and density given by (5), the background density $\rho_{\mathrm{FL}}$ is the same as that obtained by integrating over constant time slices.

$$
\begin{aligned}
\langle\rho\rangle_{t_{0}, r_{\Sigma}} & =\left(\int_{0}^{2 \pi} \int_{0}^{\pi} \int_{0}^{r_{\Sigma}} \rho \sqrt{{ }^{3} g} d r d \theta d \Phi\right) /\left(\int_{0}^{2 \pi} \int_{0}^{\pi} \int_{0}^{r_{\Sigma}} \sqrt{{ }^{3} g} d r d \theta d \Phi\right) \\
& =\left(\int_{0}^{r_{\Sigma}} \frac{M^{\prime}}{R^{2} R^{\prime}} \sqrt{R^{\prime 2} R^{4}} d r\right) /\left(4 \pi \int_{0}^{r_{\Sigma}} \sqrt{R^{\prime 2} R^{4}} d r\right) \\
& =\frac{3}{4 \pi} \frac{M\left(r_{\Sigma}\right)}{\left[R\left(r_{\Sigma}, t_{0}\right)\right]^{3}} \\
& =\frac{1}{6 \pi\left(t_{0}-t_{B}\left(r_{\Sigma}\right)\right)^{2}}=\rho_{\mathrm{FL}}
\end{aligned}
$$

where equation (3) was used.

One important point that must be made here is that a covariant averaging procedure does not exist as yet. We have used here an averaging method which is 'natural' for the comoving synchronous coordinates which lead to a $3+1$ foliation of spacetime. However, the same model in different (for example observational) coordinates would suggest a different averaging procedure which could conceivably yield different results. Therefore the claim [4] that the wavefront areas obtained in the inhomogeneous model and the averaged model are the same already seems highly unlikely.

\section{RESULTS}

We use geometric units such that $G=c=1$. If we choose a unit of time $T_{G}$ seconds to be 1 geometric time unit (gtu), then the geometric units of length, mass, density, etc. are fixed by 1 glu $=L_{G}=c T_{G}$ metres, 1 gmu= $M_{G}=$ $\left(c^{3} / G\right) T_{G} \mathrm{~kg}, 1 \mathrm{gmu} g l u^{-3}=\rho_{G}=(1 / G) T_{G}^{-2} \mathrm{~kg} \mathrm{~m}^{-3}$. For the purposes of this paper, we want units suitable to cosmological scales, so we specify that one cosmological time unit, 1 ctu, is ten billion years - of the order of the age of the universe. This gives us

\section{Cosmological Geometric Units}

\begin{tabular}{|l|l|l|l|l|}
\hline & Time & Length & Mass & Density \\
\hline Cosmological & 1 ctu & $1 \mathrm{clu}$ & $1 \mathrm{cmu}$ & $1 \mathrm{cmu} \mathrm{clu}^{-3}$ \\
\hline SI & $3.156 \times 10^{17} \mathrm{~s}$ & $9.461 \times 10^{25} \mathrm{~m}$ & $1.275 \times 10^{53} \mathrm{~kg}$ & $1.505 \times 10^{-25} \mathrm{~kg} / \mathrm{m}^{3}$ \\
\hline Astronomical & $10 \mathrm{Gyr}$ & $3.066 \mathrm{Gpc}$ & $6.409 \times 10^{22} \mathrm{M}_{\odot}$ & $1.505 \times 10^{-28} \mathrm{~g} / \mathrm{cc}$ \\
\hline
\end{tabular}

The first subsection (IIIB) gives a very simple model which satisfies the criteria for a reasonable cosmological model (with the classical Copernican principle dropped) and which provides a proof that there exist physically reasonable density behaviours which lead to a nonzero magnification or shrinking. It is obvious that averaging over the sky will not remove this effect since the model is already spherically symmetric. The second model (section IIIC) does the same, but is smoother at the origin and displays interesting behaviour in redshift space, and the third model (section IIID) is exactly homogeneous beyond a given comoving radius. 


\section{A. Form of Perturbation and General Results}

It is not easy to choose a form of area distance function for the inhomogeneous model which results in reasonable physical behaviour. So instead we choose it in the form of a 'perturbation' from a flat Friedmann model; that is,

$$
\hat{R}(r)=\hat{R}_{\mathrm{FL}}^{u}(r)(1+\delta(r))
$$

where, from (27), $\hat{R}_{\mathrm{FL}}^{u}(r)=3\left[t_{u}{ }^{1 / 3}-\left(t_{u}-r\right)^{1 / 3}\right]\left(t_{u}-r\right)^{2 / 3}$ is the area function of an underlying FL model of age $t_{1}=t_{u}$. (This 'underlying' FL model is a mathematical device with no physical significance. It can not be considered a background or average model since we have not restricted $\delta(r)$ to average out to zero in any sense.) In principle, one should choose a density function and then determine the area distance function from it or risk the possibility of assuming the result. However, if we can show that the above choice of $\hat{R}$ leads to a density profile with reasonable physical behaviour, this would suffice — since if we had initially chosen that density function, it would lead to an $\hat{R}$ as chosen above. We will show that this is indeed the case and also indicate that the model is free of shell crossings. ${ }^{8}$

Obviously, for $\delta(r)$ smooth and finite, $\hat{R}(r)$ is zero at the same places as $\hat{R}_{\mathrm{FL}}^{u}(r)$, i.e. at $r=0$ and at $r=t_{u}$. For this form of perturbation, in terms of the convenient parametrisation $v=r / t_{u}$, we find

$$
\begin{aligned}
X & \equiv\left[\frac{1}{(1-v)^{1 / 3}}-1\right] \\
M & =\frac{3}{2} t_{u} X(1-v)(1+\delta)\left[2 X(1+\delta)-\delta-3 t_{u} X(1-v) \delta^{\prime}\right]^{2} \\
\tau & =\frac{2 t_{u} X(1-v)(1+\delta)}{\left[2 X(1+\delta)-\delta-3 t_{u} X(1-v) \delta^{\prime}\right]} \\
t_{B} & =t_{0}-r-\tau \\
8 \pi \hat{\rho} & =\frac{2 X(1+\delta)-\delta-3 t_{u} X(1-v) \delta^{\prime}}{9\left[t_{u} X(1-v)(1+\delta)\right]^{2}}\left\{2 X(3+4 \delta)(1+\delta)-\delta(1+\delta)-3 t_{u} X(1-v)(5+6 \delta) \delta^{\prime}\right. \\
& \left.+36 t_{u} X^{2}(1-v)(1+\delta) \delta^{\prime}-9 t_{u}{ }^{2} X^{2}(1-v)^{2}\left[2(1+\delta) \delta^{\prime \prime}+\delta^{\prime 2}\right]\right\} \\
\frac{d \ln (1+z)}{d z} & =\left\{4 X(1+2 \delta)(1+\delta)-\delta^{2}-6 t_{u} X(1-v)(2+3 \delta) \delta^{\prime}\right. \\
& \left.+36 t_{u} X^{2}(1-v)(1+\delta) \delta^{\prime}-9 t_{u}{ }^{2} X^{2}(1-v)^{2}\left[2(1+\delta) \delta^{\prime \prime}+\delta^{\prime 2}\right]\right\} /\left[6 t_{u} X(1-v)(1+\delta)\right]
\end{aligned}
$$

If $\delta(0) \neq 0$ we find the unphysical limits $\tau(0)=0$ and $\hat{\rho}(0)=\infty$. Thus we set $\delta(0)=0$, obtaining the following limiting values:

$$
\begin{aligned}
M(0) & =\left.\frac{2\left(1-3 t_{u} \delta^{\prime}(r)\right)^{2} r^{3}}{9 t_{u}{ }^{2}}\right|_{r \rightarrow 0}=0 \\
\tau(0) & =\frac{t_{u}}{1-3 t_{u} \delta^{\prime}(0)} \\
8 \pi \hat{\rho}(0) & =\frac{4\left(1-3 t_{u} \delta^{\prime}(0)\right)^{2}}{3 t_{u}{ }^{2}} \\
\left.\frac{d \ln (1+z)}{d r}\right|_{r=0} & =\frac{2\left(1-3 t_{u} \delta^{\prime}(0)\right)}{3 t_{u}}
\end{aligned}
$$

and

$$
\begin{aligned}
M\left(t_{u}\right) & =6 t_{u}\left(1+\delta\left(t_{u}\right)\right)^{3} \\
\tau\left(t_{u}\right) & =\left.\left(t_{u}-r\right)\right|_{r \rightarrow t_{u}}=0 \\
8 \pi \hat{\rho}\left(t_{u}\right) & =\left.\frac{4(3+4 \delta(r))}{9\left(t_{u}-r\right)^{2}}\right|_{r \rightarrow t_{u}}=\infty
\end{aligned}
$$

\footnotetext{
${ }^{8}$ The necessary and sufficient conditions for there to be no shell crossings anywhere or at any time in the evolution of a flat model with $R^{\prime}>0$ are that $M(r)$ be an increasing and $t_{B}(r)$ a decreasing function. They were found (for all LTB spacetimes) by Hellaby and Lake 15 .
} 


$$
\left.\frac{d \ln (1+z)}{d r}\right|_{t_{u}}=\left.\frac{2(1+2 \delta(r))}{3\left(t_{u}-r\right)}\right|_{r \rightarrow t_{u}}=\infty
$$

(The limits for the background FL model are obtained by setting $\delta=0=\delta^{\prime}=\delta^{\prime \prime}$ and replacing $t_{u}$ by $t_{b}$.) From numerical experimentation we concluded, in order to avoid shell crossings, that $\delta(r)$ must remain sufficiently far away from zero over most if not the entire range of $r>0$, and certainly near $r=t_{u}$. We want the proper time from the bang surface to the null surface on the central worldine to be the 'true' age of the universe; that is, we want it to be $t_{0}$, the time at the origin of the LTB model. By setting $\tau(0)=t_{0}$ in (43), the age of the underlying model is determined

$$
t_{u}=t_{0}\left(1-3 t_{1} \delta^{\prime}(0)\right) .
$$

The parameter $t_{u}$ is the $r$-coordinate value at which the null cone of the LTB model intersects the bang. We will average quantities on this scale; that is to say, we shall take $r_{\Sigma}=t_{u}$. We match this inhomogeneous universe to a flat FL model at the surface $r_{\Sigma}$ by equating the masses and bang times at that point. This then determines the time

$t_{1}=t_{b}$ in the background FL model which we will use for our comparison. At $r=t_{u}, \hat{R}=0$ and (46) shows that at this point,

$$
M\left(t_{u}\right)=M_{\mathrm{FL}}^{u}\left(t_{u}\right)\left(1+\delta\left(t_{u}\right)\right)^{3}=6 t_{u}\left(1+\delta\left(t_{u}\right)\right)^{3} .
$$

In the background FL model the value of the mass at $\Sigma$ is $6 t_{b}$ and this is what we have to match the inhomogeneous mass to. This gives us a value of the age for the background flat FL model of

$$
t_{b}=t_{u}\left(1+\delta\left(t_{u}\right)\right)^{3} .
$$

\section{B. A Regular Model which Exhibits Shrinking and Magnification}

The following simple example is physically well behaved, being free of shell crossings at all times in its evolution for $r \leq t_{u}$. Since $t_{B}^{\prime} \neq 0$ at the origin, the model is not as smooth there as one would like, but there are no physical problems. We choose $\delta(r)$ for our first model, LTB1, to be

$$
\delta(r)=-\frac{1}{5} \sin \left(\frac{0.8 \pi r}{t_{u}}\right) .
$$

When we set $\tau(0)=t_{0}=1$ then

$$
t_{u}=t_{0}\left(1+\frac{12 \pi}{25}\right)
$$

and the age for the background model FL1, after matching the masses, is

$$
t_{b}=t_{u}\left(1-\frac{\sin 0.8 \pi}{5}\right)^{3}
$$

The calculation of the redshift was done by a numerical quadrature of (23). Figs. 1 and 2 compare the dependence of area distance and density on redshift, $\hat{R}(z) \& \rho \hat{(z)}$, in LTB1 with the corresponding functions in the background model FL1. 


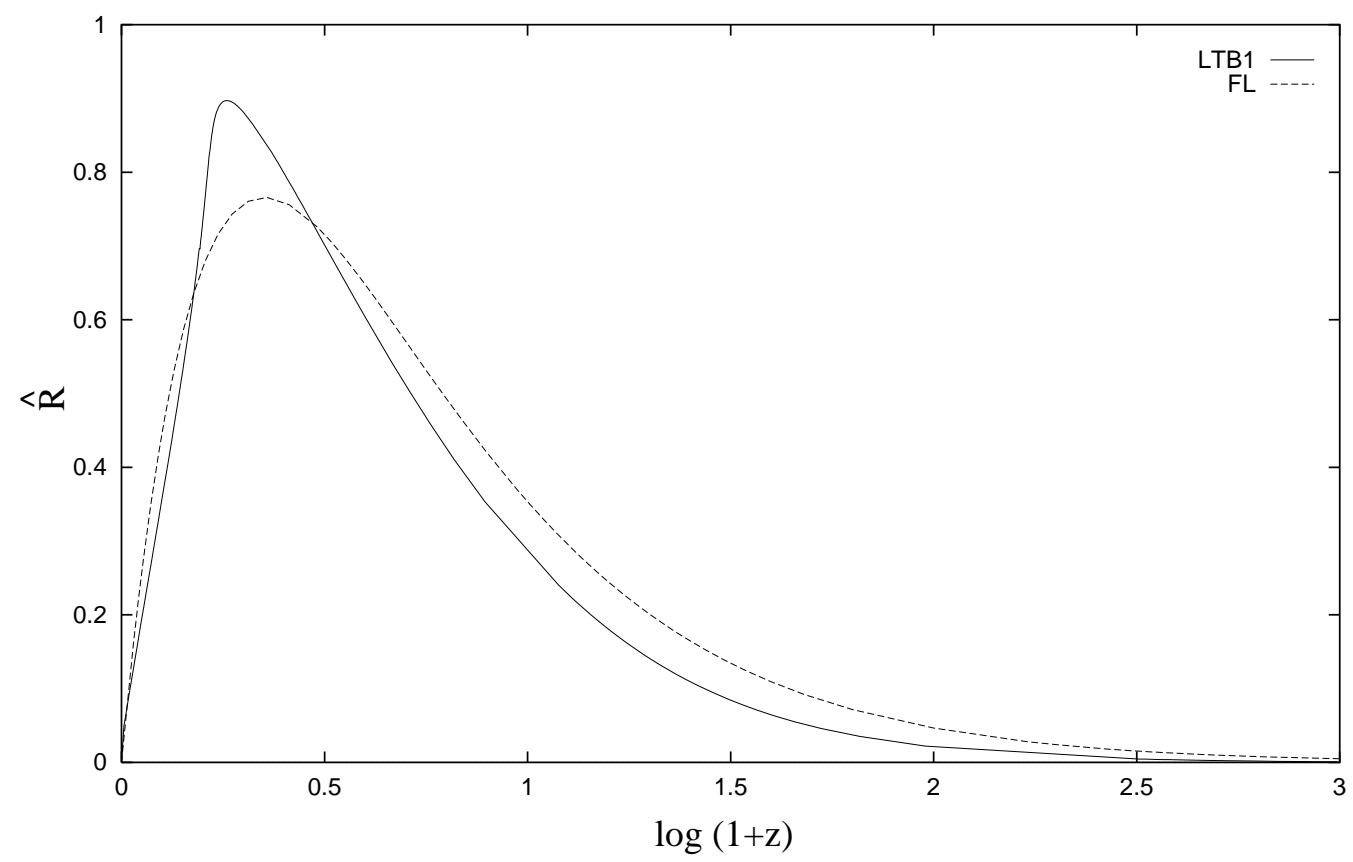

FIG. 1. A plot of area distance against redshift $\hat{R}(z) \& \hat{R}_{F L}(z)$ on the past null cone of the inhomogeneous model LTB1 and the corresponding background model FL1. The units of $\hat{R}$ are cosmological length units, and all the figures use base 10 logs. This shows that there are systematic shrinking $\left(\hat{R}>\hat{R}_{F L}\right)$ and magnification $\left(\hat{R}<\hat{R}_{F L}\right)$ effects due to purely radial lensing, which obviously cannot be removed by averaging over large angular scales or even the whole sky. Effects of true lensing in a more realistic universe would be imposed on top of this.

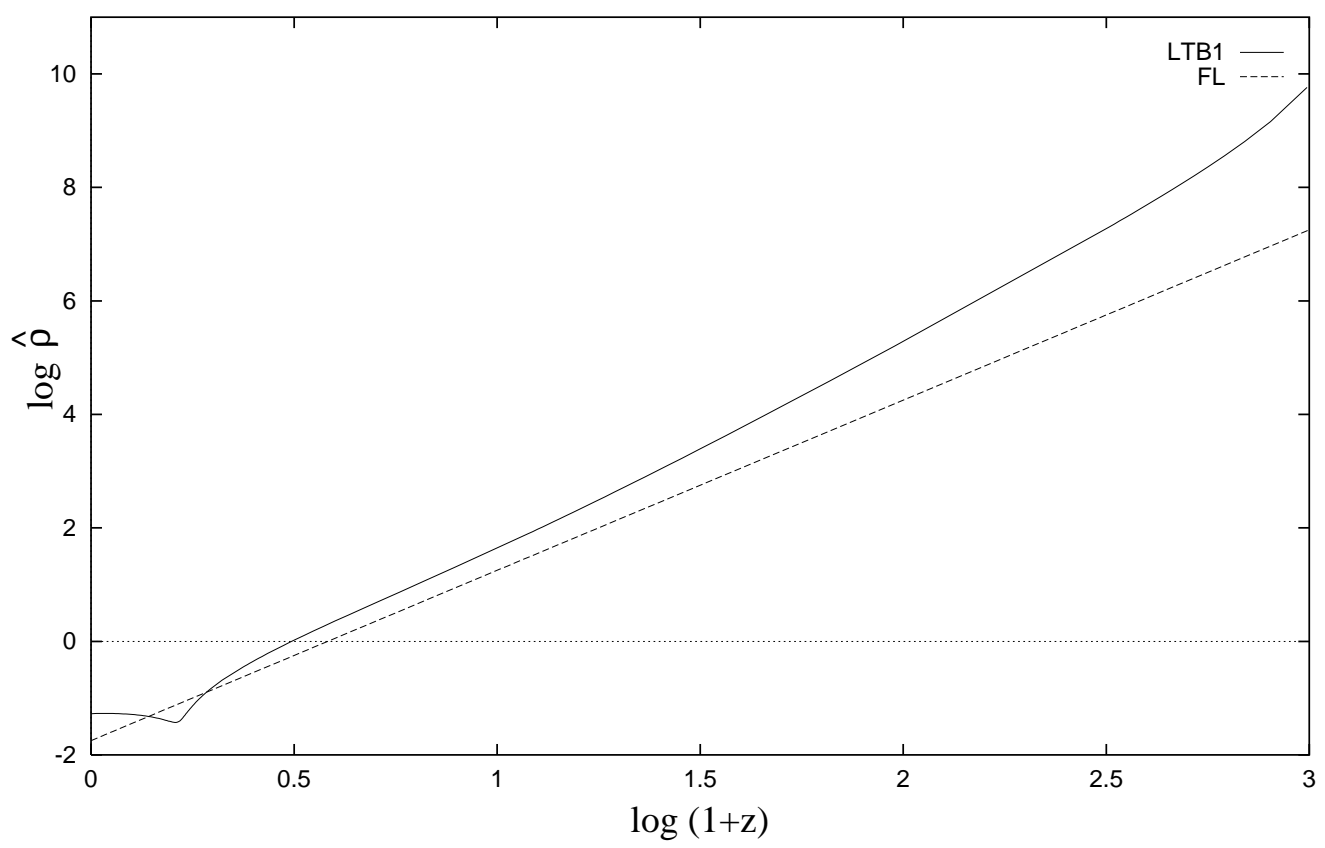

FIG. 2. The density of matter $\hat{\rho}(z) \& \hat{\rho}_{F L}(z)$ against redshift on the past null cone in the models of FIG. 1, LTB1 and its corresponding background model FL1. The units are cosmological density units $\left(\mathrm{cmu}^{\mathrm{cl}} \mathrm{u}^{-3}\right)$. When comparing with FIG. 1, we see that roughly speaking, magnification occurs for obects in or just beyond an overdense region, and shrinking occurs for objects in or just beyond an underdensity. 
It is important to plot these quantities in terms of the observable quantity $z$ for two reasons. First of all, in the transformation $r \rightarrow z$, the possibility exists that the area distances of the flat and inhomogeneous models might transform into each other. Secondly, under certain circumstances the redshift becomes disordered with distance and unexpected behaviour might occur, as the following model illustrates.

\section{A Regular Model with Multivalued Observations}

As an illustration of how different the physical quantities plotted against radial coordinate $r$ as opposed to those same quantities plotted against redshift $z$ may appear, we present here an LTB model for which the redshift becomes disordered with distance at some points and then ordered again at later points.

The universe is chosen as above but with a 'perturbation function' of

$$
\delta(r)=-\frac{1}{4} \sin \left(\frac{0.75 \pi r}{t_{u}}\right)-\frac{1}{4^{6}}\left(\frac{11}{\pi}+\frac{3}{2}\right)\left[1-\cos \left(\frac{4 \pi r}{t_{u}}\right)\right] .
$$

This model, which we call LTB2, is also free of shell crossings at any time for $r \leq t_{u}$ and has a completely smooth and regular origin (where $t_{B}^{\prime}=0$ ). Setting $\tau(0)=t_{0}=1$ once again gives

$$
t_{u}=t_{0}\left(1+\frac{9 \pi}{16}\right)
$$

and the appropriate background model FL2 has age

$$
t_{b}=t_{u}\left(1-\frac{\sin 0.75 \pi}{4}\right)^{3} .
$$

which are different from those of LTB1. Figs. 3 의 show $\hat{R}$ and $\hat{\rho}$ against $r$ and against $z$ for LTB2 and FL2.

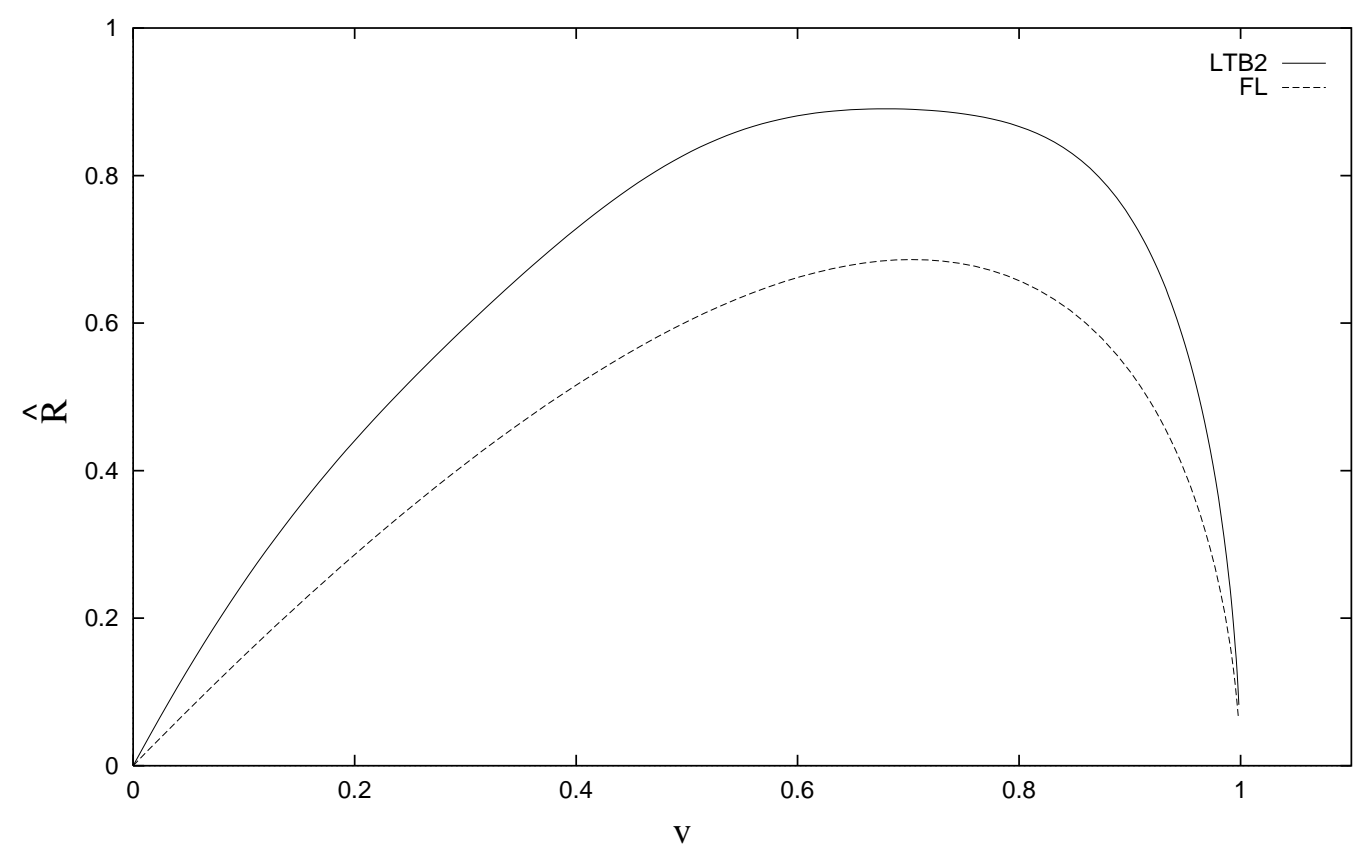

FIG. 3. The area distance functions $\hat{R}(r) \& \hat{R}_{F L}(r)$ on the past null cones of the second model, LTB2, and its corresponding background model, FL2, given in cosmological units. The horizontal variable is $v=r / t_{u}$ or $r / t_{b}$ for LTB2 and FL2 respectively. The physical behaviour of LTB2 as plotted against $v$ appears normal. 


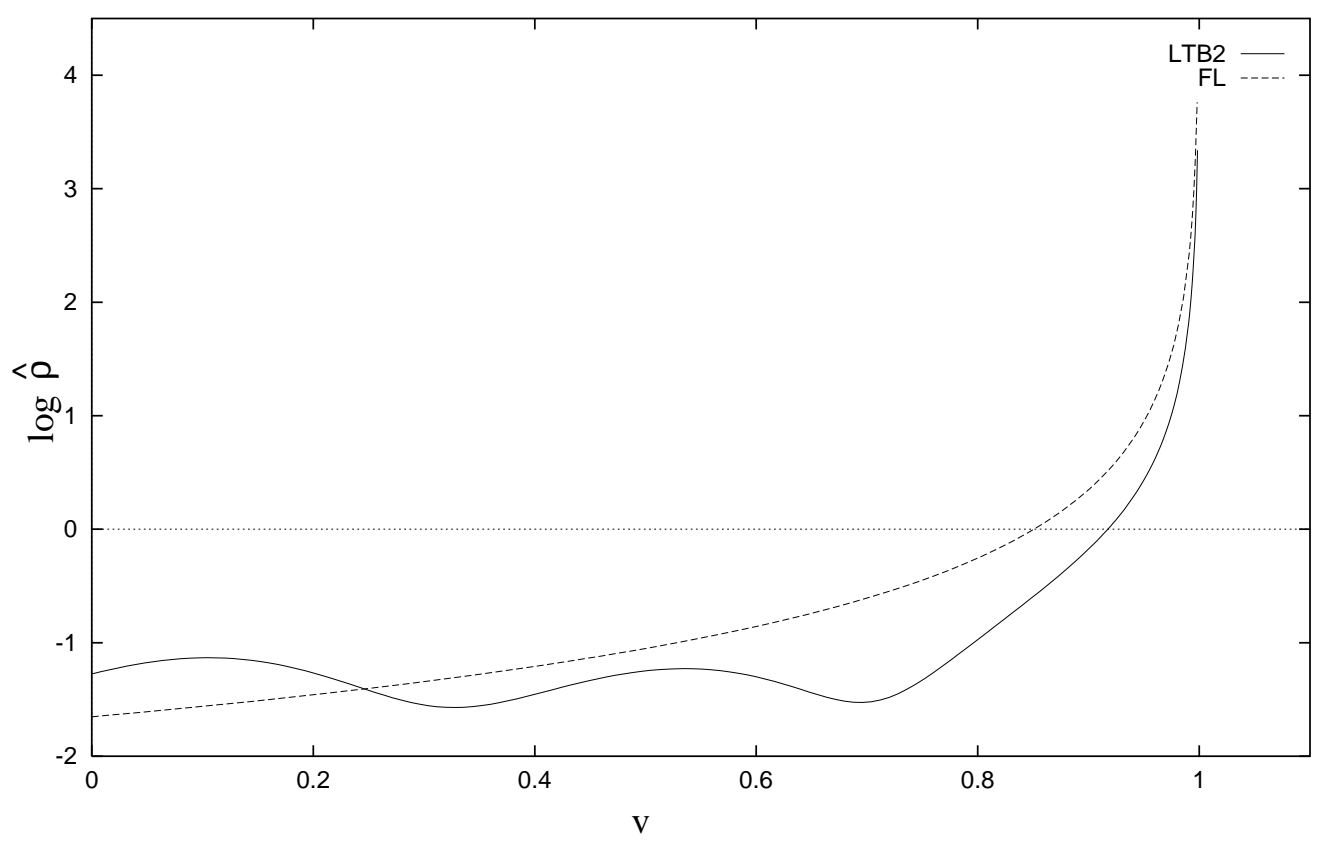

FIG. 4. The densities on the past null cone for LTB2 and FL2, $\hat{\rho}(r)$ and $\hat{\rho}_{F L}(r)$, in cosmological units. Again, LTB2's inhomogeneous profile vs $v$ appears quite acceptable.

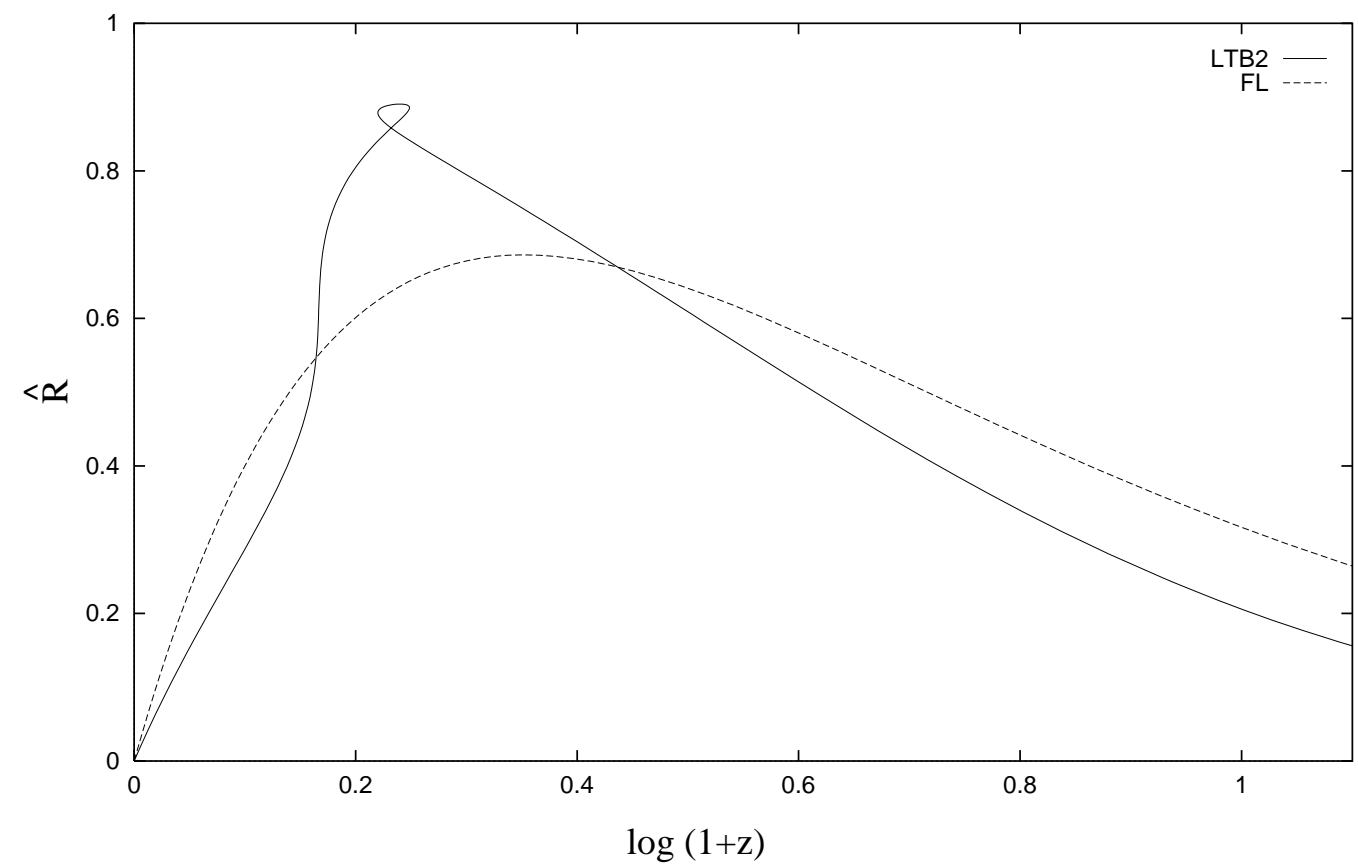

FIG. 5. Area distance against redshift $\hat{R}(z) \& \hat{R}_{F L}(z)$ for LTB2 and FL2. The interesting point to note is that at some redshifts the area distance in LTB2 is multivalued. For $\log _{10}(1+z) \geq 1$ the graph looks very much like FIG. 11. 


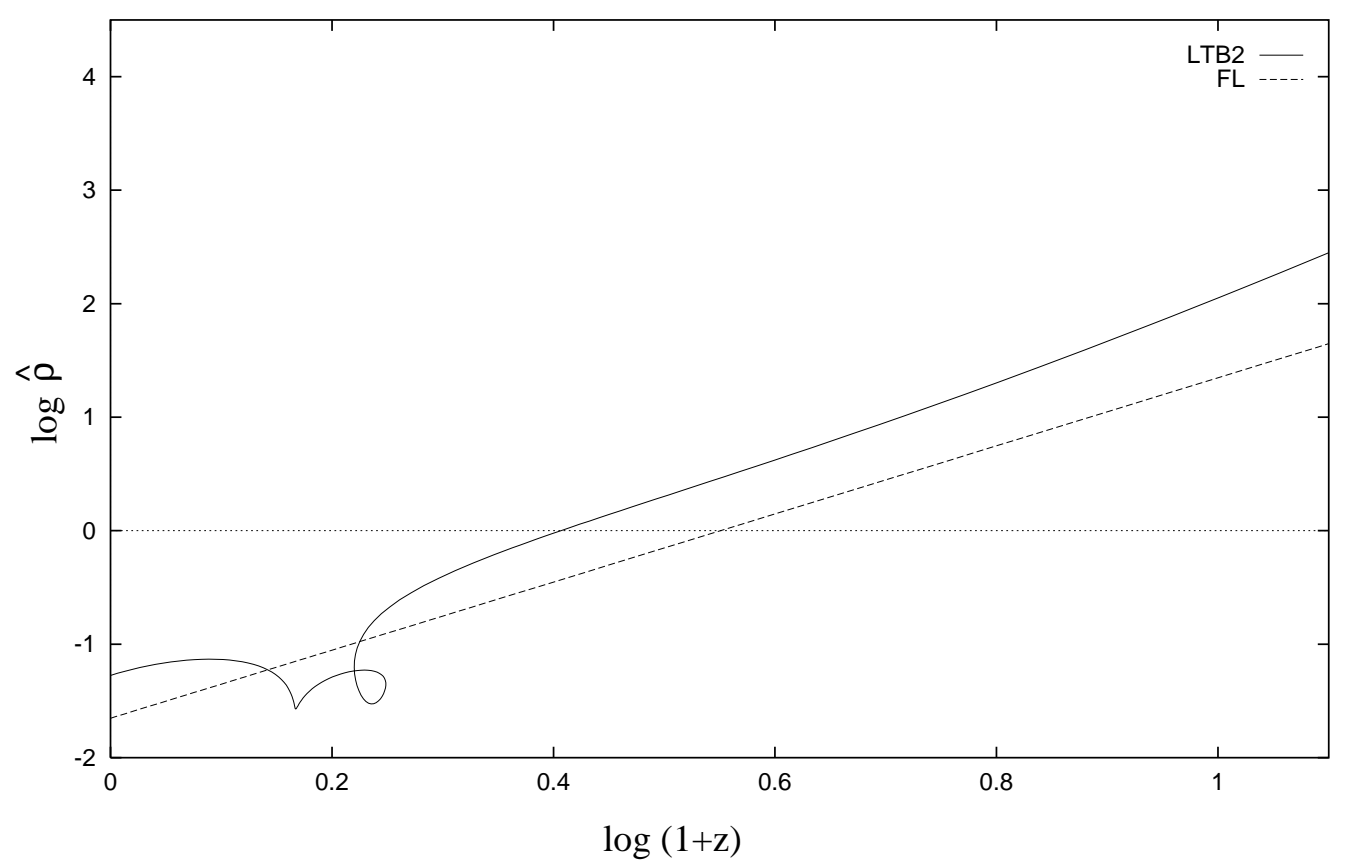

FIG. 6. The densities, $\hat{\rho}(z)$ and $\hat{\rho}_{F L}(z)$, against redshift for LTB2 and FL2. Note the quaint 'looping' behaviour. For $\log _{10}(1+z) \geq 1$ the graph looks very much like FIG. 2 .

This model provides a good illustration of why one has to be careful in ascribing physical behaviour in a certain coordinate system. Viewed as functions of $r, \hat{R}$ and $\hat{\rho}$ have fairly standard behaviour, but viewed in terms of the observable quantity $z$, the density and area distance become multivalued. Hence, three objects with the same intrinsic luminosity located at different distances appear at the same $z$, with three different apparent luminosities (or area distances).

Our numerical experiments indicate that the redshift on the light cone is most sensitive to perturbations in the vicinity of the maximum in $\hat{R}(z)$. All our models in which $d z / d r$ became negative did so in this region. The looping behaviour in the $\hat{R}$ vs $\log (1+z)$ plot occurs when the maximum and minimum in the $\log (1+z)$ vs $r$ graph bracket the maximum in the $\hat{R}$ vs $r$ graph. Similarly, perturbations more easily generate a maximum and minimum in the $\hat{\rho}(z)$ near the maximum in $\hat{R}$, hence the loop in that graph.

\section{A Swiss-Cheese Type Model}

In order to emphasise the effect of inhomogeneity on the past null cone, we consider a model which is homogeneous on the large scale. We construct a local inhomogeneity which is properly matched onto an exact FL model at some comoving radius, $r=r_{j}$, somewhere between us and the particle horizon. In other words, the outer regions are exactly homogeneous. This is a simple model of a universe which is FL on some averaging scale less than the horizon size, and is seen by an observer located in an overdensity.

In particular, for LTB3, we set $r_{j}=0.85 t_{u}=2.352$, we retain the inhomogeneous LTB2 model for the interior, $r \leq r_{j}$, and for the exterior, $r \geq r_{j}$, we use the matching FL model, FLJ, with the same mass and bang time as LTB2 at the boundary $r_{j}$. Obviously the background model FL3 that we fit at the particle horizon is identically the one

\footnotetext{
${ }^{9}$ In order to make the radial coordinate continuous at this matching, we use $\hat{R}_{F L J}=3\left[t_{3}^{1 / 3}-\left(t_{2}-r\right)^{1 / 3}\right]\left(t_{2}-r\right)^{2 / 3}, M_{F L J}=$ $6\left[t_{3}^{1 / 3}-\left(t_{2}-r\right)^{1 / 3}\right]^{3}$, where $t_{2}=\tau_{L T B}\left(r_{j}\right)+r_{j}$ and $t_{3}^{1 / 3}=\tau_{L T B}^{1 / 3}\left(r_{j}\right)+\left(M_{L T B}\left(r_{j}\right) / 6\right)^{1 / 3}$. These differ from the purely FL
} 
matched at $r_{j}$, FLJ, but written in the form (26)-(29) - i.e. with a different $r$ coordinate.

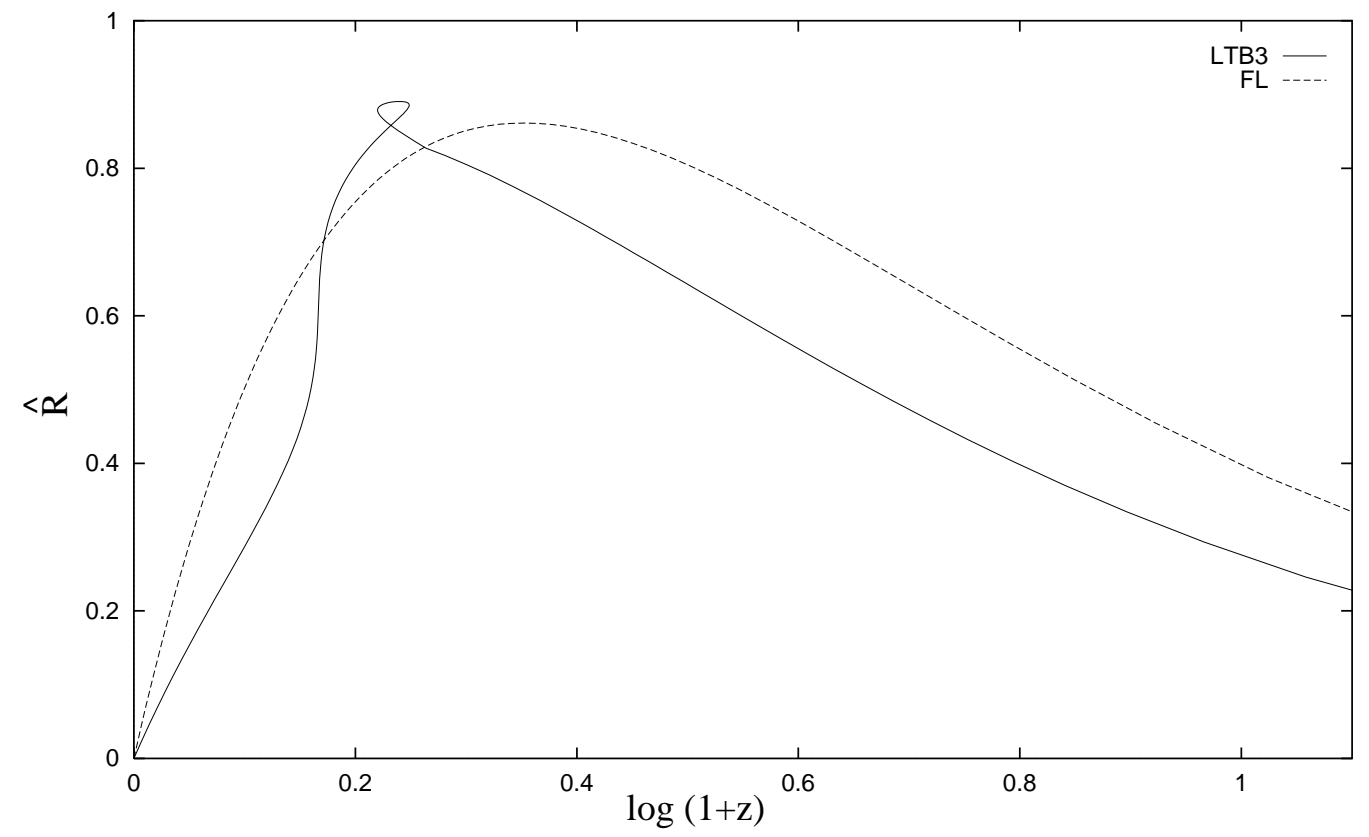

FIG. 7. Area distance against redshift $\hat{R}(z) \& \hat{R}_{F L}(z)$ for the swiss-cheese model LTB3 and its corresponding background model FL3. The boundary is located at $\log _{10}\left(1+z_{j}\right)=0.2635$, with the model being identical to LTB2 for smaller $z$, and exactly FL for larger $z$. Though the background and matched FL models are identical, the $\hat{R}(z)$ curves do not match up in the exterior region - there is a constant displacement parallel to the $\log (1+z)$ axis. This emphasises the fact that the $z$ integral is path dependent, and so the value of $z$ at any given area distance is affected by the warping of the null cone in the inhomogeneous interior.

expressions because our definition of $r$ is also path dependent. It turns out that $t_{b}=t_{3}$. 


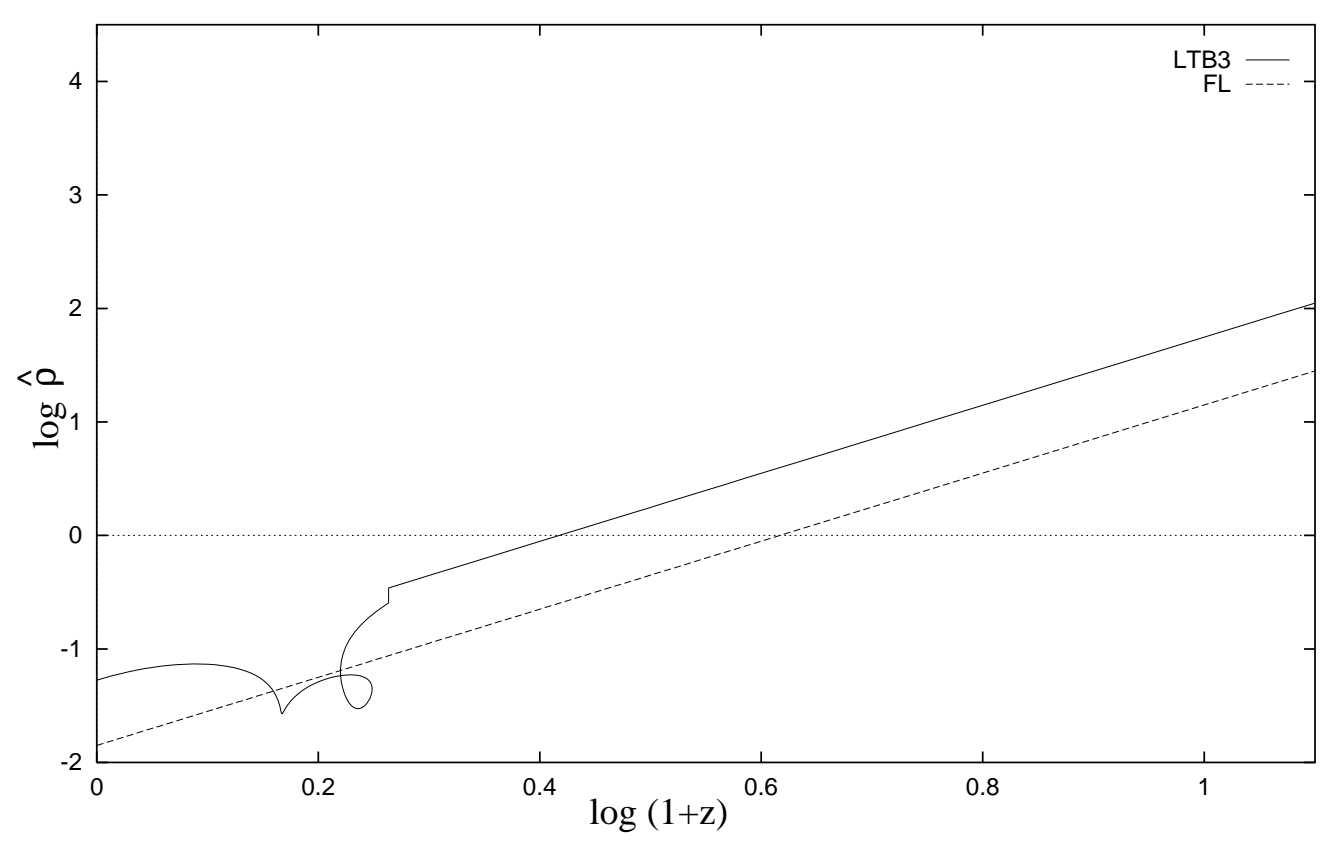

FIG. 8. Density against redshift $\hat{\rho}(z) \& \hat{\rho}_{F L}(z)$ for the swiss-cheese model LTB3 and its background model FL3. The density jumps at the boundary, $\log _{10}\left(1+z_{j}\right)=0.2635$, but this is not unrealistic. (Within the constraints of a parabolic model, it could only have been made continuous at the expense of creating shell crossings.) As with the area distance, the two $\hat{\rho}(z)$ curves do not co-incide in the FL exterior, because the shape of the null cone is determined by the spacetime geometry it encounters along its path. The boundary could have been put at any $z$ value, and it is evident from FIGs. 5 and $\mathrm{f}$ especially that in general the standard FL relations are not recovered in the FL exterior.

Figs 17 and 8 show clearly that the null cone relations $\hat{R}(z)$ and $\hat{\rho}(z)$ in this model (LTB3) do not return to the standard FL ones in the FL region beyond the boundary, $z>z_{j}$. This is because the redshift $z$ is path dependent and the inhomogeneous interior distorts the null cone. After passing through the inhomogeneity, the null cone, and thus the value of $\log _{10}(1+z)$, are displaced relative to a model which is FL throughout. This holds generally for any choice of inhomogeneous interior and boundary position.

\section{CONCLUSIONS}

The general belief that photon conservation implies that the total area of an incoming wavefront must be the same as in the background, matter-averaged, FL model is contradicted by our results. The functions $\hat{R}(z)$ and $\hat{\rho}(z)$ in an inhomogeneous model differ from their standard FL forms, because the redshift $z$ is path dependent and the null cone is warped. Thus, even if the inhomogeneous model is exactly homogeneous beyond some radius, the standard FL forms are not recovered. This means that a universe which is FL on average will not in general present standard FL forms of $\hat{R}(z)$ and $\hat{\rho}(z)$ to an observer.

The spherically symmetric model used here is simple but effective, since averaging over direction cannot change the results. In more realistic models of the lumpy universe this effect will still be present, and we expect full gravitational lensing to occur, resulting in more significant deviations from the FL formula.

This investigation used a parabolic LTB model, where the areal radius $\hat{R}$ is also the area distance of the 2-sphere wavefronts of the past null cone. The density in the LTB model is averaged to give a background Einstein-de Sitter $(\Omega=1)$ model, and it is tested against this model. Although there exists no covariant way to perform this averaging, we use the 'natural' one defined by the use of junction conditions, here equivalent to the one used in astrophysical problems: that is, averaging by integrating the rest mass and proper volume on constant time slices. More importantly, the functions $\hat{R}(z)$ and $\hat{\rho}(z)$ in the examples are obviously perturbed away from the standard FL ones, so that no one FL model can give the same $\hat{R}$ and $\hat{\rho}$ values as an inhomogeneous one at a variety of $z$ values.

The results show that it is quite easy to have areas in the inhomogeneous models which differ significantly from 
areas in the background, matter-averaged FL model. The result may either be shrinking (the background FL area distance underestimates the real area distance at that redshift) or magnification (background FL area distance is an overestimate). The presence of loops in the $\hat{R}-z$ and $\hat{\rho}$ - $z$ graphs is analogous to the well known 'finger of God' effect familiar in redshift maps of the galaxy distribution.

Our results and conclusions generally agree with those of Kurki-Suonio and Liang [12], who calculated obervational relations numerically in 4 hyperbolic LTB models out to $z=0.5$. They generated mild and strong deviations from the FL observational relations, with 'bang time' inhomogeneities and 'areal radius' inhomogeneities having opposite effects on the oberved matter distribution in redshift space, compared with the present day distribution. Their method did not permit the redshift to be disordered with distance.

Whilst the major aim of this paper has been the above thesis, the choice of radial coordinate which locates the observer's null cone will be of use in future analyses of observations in these isotropic dust models.

An important caveat is that since the LTB model does not allow for formation of caustics in the null cone of the central observer, it cannot be considered a useful model for obtaining quantitative 'real world' results. Rather this paper should be viewed as a proof that even purely radial lensing distorts the area distance-redshift relation significantly. If the observer moves away from the central position, then continuity ensures that the radial effects found here will still be present, and the effects of true lensing will be superimposed. As argued in [1.21, we expect caustics to skew the area towards larger values, so that most objects in the universe are demagnified.

The importance of all this is that it opens up the way for considering the effects of lensing by inhomogeneities on large angular-scale number counts and area distances as opposed to limiting discussion to lensing effects on small scales.

[1] G.F.R. Ellis, B.A.C.C. Bassett and P.K.S. Dunsby, submitted to, Class. Q. Grav. (1997) .

[2] D.M. Solomons, G.F.R. Ellis and P. Haines submitted to Class. Q. Grav. (1997).

[3] C.C. Dyer and R.C. Roeder, Astrophys. J., 174, L115, (1972).

[4] S. Weinberg, Astrophys. J., 208, L1-3, (1976).

[5] P. Schneider, J. Ehlers and E.E. Falco, Gravitational Lenses, (Berlin: Springer Verlag), (1992).

[6] E.V. Linder, Astron. and Astrophys., 206, 190, (1988).

[7] G. Lemaître, Ann. Soc. Scient. Bruxelles, A53, 51, (1933).

[8] R.C. Tolman, Proc. Nat. Acad. Sci., 20, 169, (1934).

[9] H. Bondi, Mon. Not. Roy. Astron. Soc., 107, 410, (1947).

[10] R. Maartens, N.P. Humphreys, D.R. Matravers and W. Stoeger, Class. Q. Grav. 13, 253-264, (1996). (gr-qc/9511045).

[11] N.P. Humphreys, R. Maartens and D.R. Matravers, Portsmouth University preprint RCG 96/1, (1996).

[12] H. Kurki-Suonio and E. Liang, Astrophys. J., 390, 5-16 (1992).

[13] G. Darmois, Mémorial des Sciences Mathematiques, Fasc.25, Gaulthier-Villars, Paris, (1927).

[14] W. Israel, Il Nuovo Cim.. 44B, 1 (1966), and errata in ibid 48B, 463, (1967).

[15] C. Hellaby and K. Lake, Astrophys. J., 290, 381-9, (1985), and errata in ibid 300, 461, (1986). 